\section{Late Presentation of Congenital Diaphragmatic Hernia in a 3 Month-Old Infant}

\section{Abstract}

A 3-month-old female infant presented with vomiting for 1-month, poor feeding and lethargy for few days with history of fever at home. On examination was noted to be lethargic, hypoxic, tachypnoeic with recessions and poor air entry and cold extremities. She was resuscitated with oxygen and fluids and was being treated for suspected sepsis. A chest X-ray (CXR) showed bowel loops in the left hemi thorax confirming the diagnosis of congenital diaphragmatic hernia (CDH). The infant was subsequently transferred to a tertiary centre where the defect was surgically corrected. This case report highlights the difficulty in suspecting and diagnosing late presenting $\mathrm{CDH}$ after the neonatal period.

Keywords: Hernias; Diaphragmatic; Congenital
Gowai $\mathrm{H}^{1 *}$ and Kanagasabapathy $\mathrm{S}^{2}$

\author{
1 Department of General Paediatrics, \\ Doncaster Royal infirmary, Doncaster, UK \\ 2 Department of Paediatrics, Rotherham \\ General Hospital, Rotherham, UK
} *Corresponding author:
Hani Ahmed Gowai

hani4u2c@hotmail.com

Department of General Paediatrics, Doncaster Royal Infirmary, Doncaster, UK.

Tel: +447426965359

Citation: Gowai H, Kanagasabapathy S (2020) Late Presentation of Congenital Diaphragmatic Hernia in a 3 Month-Old Infant. Pediatr Emerg Care Med Open Access Vol.5 No.1:2
A 3-month-old girl presented to the accident and emergency (A\&E) department (referred by their GP) with worsening vomiting over a month that was initially milky and later became slightly green stained. She was also less active, feeding poorly and was felt to be hot at home. She was born at term after an uneventful pregnancy with normal antenatal scans at 20 weeks of gestation and no family history of significance. 4 weeks prior to this presentation, she was seen at her local hospital with vomiting that was thought to be due to mild reflux as she was growing and was discharged home without medications. On arrival to $A \& E$, her observations were: Temperature: $36.3^{\circ} \mathrm{C}$, Heart rate: $180 / \mathrm{min}$, capillary refill time 4 seconds, Respiratory rate: $60 / \mathrm{min}$, oxygen saturation $\left(\mathrm{SPO}_{2}\right) 85 \%$ in air. She also had moderate intercostal recessions and poor air entry bilaterally. She was put on $15 \mathrm{~L} / \mathrm{min}$ $\mathrm{O}_{2}$ via face mask and her $\mathrm{SPO}_{2}$ improved to $98 \%$ and was placed on a trans-warmer. A peripheral iv cannula was sited, blood samples were taken for culture, $C$ reactive protein (CRP), liver function tests (LFT), urea and electrolytes (U/Es) and blood gas. A fluid bolus of $20 \mathrm{ml} / \mathrm{kg}$ of 0.9 sodium chloride was administered and a CXR was requested. She was being treated for suspected sepsis and a dose of IV antibiotic was administered. Her CXR** showed bowel loops within the left hemi thorax consistent with a diagnosis of $\mathrm{CDH}$. She was transferred to a tertiary paediatric surgical centre where she underwent a corrective procedure and had smooth recovery afterwards.

\section{Discussion}

$\mathrm{CDH}$ is an anomaly with an incidence (that varies based on the population being studied) of about 2.7 per 10000 live birth [1]. It implies a defect in the diaphragm through which abdominal viscera can herniate into the chest. It is often diagnosed antenatally on routine ultrasonography scans with varying detection rates in different centres [2], but a number of cases also present later after birth and these can usually pose a challenge to clinicians. If it was not picked up antenatally, it can present either during early life (neonatal period) or even later. Often, respiratory distress is a common presentation during neonatal period with CXRs showing bowel loops in the chest. Beyond the neonatal period, diagnosis becomes even more difficult due to the wide range of clinical presentations of $\mathrm{CDH}$. Vomiting (as in our case), various levels of breathing difficulty (including exercise related and orthopnoea), failure to thrive, recurrent chest infections and even frank surgical abdomen have all been noted and reported [3]. The severity of presenting symptoms is influenced by the degree of lung development (i.e., pulmonary hypoplasia) and whether there is accompanying persistent pulmonary hypertension (PPHN) which will also affect the prognosis. There are a number of reasons for late or missed diagnosis of $\mathrm{CDH} 4$. 
One of the theories is that sometimes herniation occurs late in pregnancy which means early scans will miss it. Also, it is possible that the high intrathoracic pressure prevents herniation which happens later when pressure is reduced. Besides, solid organs like liver and spleen may occlude the defect to start with and later with growth the defect is exposed allowing herniation. So, a normal previous CXR, although it makes it difficult to think of, does not exclude the diagnosis of $\mathrm{CDH}$ and there are many case reports to support this [4]. Occasionally, the hernia could be intermittent. When patients are flat they become symptomatic and may have positive findings on radiographs as opposed to when they ambulate or are erect allowing the viscera to escape back to the abdominal cavity. There have been many case reports about cases of $\mathrm{CDH}$ that had their radiographs with positive signs misinterpreted. Diagnoses like lobar pneumonia, lung cysts and pneumatoceles and even pneumothoraces were sometimes made and in some occasion chest drains were inserted [4]. Having a nasogastric tube inserted before taking x-rays can be helpful especially with left side hernias where a herniated stomach can be spotted. If the diagnosis is suspected and plain films could not offer assurance, other modalities should be used like ultrasound scan; contrast studies and CT scan which will help delineate the defect and confirm diagnosis [5]. Having an antenatal diagnosis informs clinicians and helps them plan for delivery and the care after. It also provides grounds for counselling parents and managing expectations. On the contrary, it is worth mentioning that in countries and areas with low resourced settings, there is lack of routine antenatal anomaly scan services [6]. Thus, a large number of $\mathrm{CDH}$ cases are diagnosed postnatally and this depends on the high index of suspicion based on clinical grounds and using simple available tools like plain radiographs (Figure 1).

\section{References}

1 Gallot D, Boda C, Ughetto S (2007) Prenatal detection and outcome of congenital diaphragmatic hernia: a French registry-based study. Ultrasound Obstet Gynecol 29: 276-283.

2 Garne E (2002) Congenital diaphragmatic hernia: Evaluation of prenatal diagnosis in 20 European regions. Ultrasound in Obstetrics and Gynecology 19: 329-333.

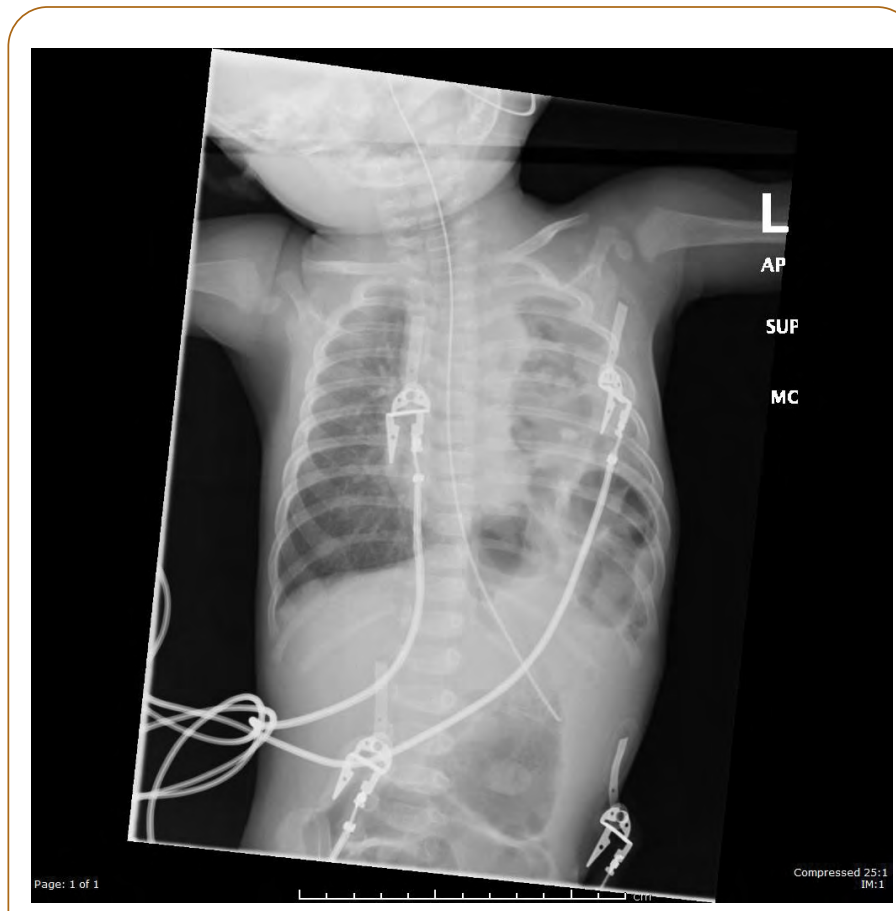

Figure 1 Image of chest X-ray.

\section{Conclusion}

If missed antenatally, the diagnosis of late presenting $\mathrm{CDH}$ can be challenging and requires a high index of suspicion. $\mathrm{CDH}$ is a diagnosis to be considered in new born infants with respiratory distress. Plain radiographs can be diagnostic if interpreted correctly, and placement of nasogastric tube before taking images is very helpful. Other modalities of investigation like ultrasound, barium and CT can help if still in doubt.

3 Berman L, Stringer D, Ein SH (1988) The late presenting paediatric Bochdalek hernia: A 20-year review. J Pediatr Surg 23:735-739.

4 Gleeson F, Spitz L (1991) Pitfalls in the diagnosis of congenital diaphragmatic hernia. Arch Dis Child 66: 670-671.

5 Oh KS, Newman B, Bender TM (1988) Radiological evaluation of the diaphragm. Radiol Clin North Am 26: 355-364.

6 Abubakar AM, Bello MA, Chinda JY (2011) Challenges in the management of early versus late presenting congenital diaphragmatic hernia in a poor resource setting. Afr J Paediatr Surg 8: 29-33. 